\title{
ON THE EXISTENCE OF NONSMOOTH CONTROL-LYAPUNOV FUNCTIONS IN THE SENSE OF GENERALIZED GRADIENTS
}

\author{
LUDOVIC RIFFORD ${ }^{1}$
}

\begin{abstract}
Let $\dot{x}=f(x, u)$ be a general control system; the existence of a smooth control-Lyapunov function does not imply the existence of a continuous stabilizing feedback. However, we show that it allows us to design a stabilizing feedback in the Krasovskii (or Filippov) sense. Moreover, we recall a definition of a control-Lyapunov function in the case of a nonsmooth function; it is based on Clarke's generalized gradient. Finally, with an inedite proof we prove that the existence of this type of controlLyapunov function is equivalent to the existence of a classical control-Lyapunov function. This property leads to a generalization of a result on the systems with integrator.
\end{abstract}

Mathematics Subject Classification. 93D05, 93D15, 93D20, 93D30, 93D09, 93B05, 34D20, 49J52, 49K24, 70K15.

Received August 21, 2000. Revised May 2, 2001.

\section{INTRODUCTION}

The object of this paper is to study the control systems of the form

$$
\dot{x}=f(x, u),
$$

which admit nice properties of stabilization at the origin. First, using the Kurzweil's result [20], it is straightforward to show that if a control system admits a continuous feedback $u: \mathbb{R}^{n} \rightarrow U$ for which the closed-loop system

$$
\dot{x}=f(x, u(x))
$$

is globally asymptotically stable at the origin, then it possesses a smooth control-Lyapunov function. The converse is false. Many systems which possess a smooth control-Lyapunov function do not admit continuous stabilizing feedbacks. However, as it was shown by Artstein [1], such systems admit relaxed continuous feedbacks. If moreover the system is affine in the control, these ones can be taken to be continuous outside the origin. Actually, the relaxed feedbacks which are provided by Artstein are not the only one which allow us to overcome the lack of continuous stabilizing closed-loops. Other kinds of feedbacks coming from notions developped by Filippov [14] and Krasovskii [19] enable us as well to stabilize correctly the systems which admit smooth control-Lyapunov functions. Considering a closed-loop system $x \mapsto f(x, u(x))$ that is not necessarly continuous, they associate with it a differential inclusion which is upper semicontinuous with convex values. Roughly

Keywords and phrases: Asymptotic stabilizability, converse Lyapunov theorem, nonsmooth analysis, differential inclusion, Filippov and Krasovskii solutions, feedback.

1 Institut Girard Desargues, Université Claude Bernard Lyon I, 69622 Villeurbanne, France;

e-mail: rifford@desargues.univ-lyon1.fr 
speaking, in the case of Krasovskii, giving a state $x_{0}$ it puts together all the possible values of the vector field $f(x, u(x))$ around $x_{0}$. The corresponding trajectories of this differential inclusion have indeed the same behavior as the perturbed trajectories associated with the discontinuous ordinary differential equation $\dot{x}=f(x, u(x))$ $($ see $[17,21])$.

On the other hand, it is not necessary to work with smooth functions. Using Clarke's generalized gradients permits us to extend the classical definition of control-Lyapunov function: we do not require them to be smooth anymore, but only locally Lipschitz. We next refer to them as control-Lyapunov functions in the sense of generalized gradients. In this way, we can give a sense to some locally Lipschitz functions which arises naturally as potential control-Lyapunov functions. We prove in this paper that the existence of a control-Lyapunov function in the sense of generalized gradients is both equivalent to existence of a smooth control-Lyapunov function and to existence of a stabilizing feedback of Krasovskii or Filippov type. Even if the first equivalence is new, we stress that the novelty of this paper is not so much in the results but in the proofs. In particular, we precise that the notion of locally Lipschitz control-Lyapunov function ever appeared in [15].

In addition, we present a precise example of stabilization problem where the use of a nonsmooth controlLyapunov function comes out naturally. We prove that if a control system possesses a locally Lipschitz stabilizing feedback, then the corresponding control system with integrator admits a stabilizing feedback which is continuous on the state space. This question concerning the integrator problem has been studied by many authors $[5,10,12,30,32]$ in the smooth case (i.e. when the dynamics is smooth); however let us notice that we can recognize the nonsmooth cases in the works of Freeman and Kokotovic [15] and Praly and Teel [31].

Our paper is organised as follows: in Section 2 we describe our results. In Section 3 we apply our main theorem to the integrator problem. In Section 4, we give the proofs of Theorems 1 and 2 and we conclude with some comments.

Throughout this paper $\langle\cdot, \cdot\rangle$ denotes the inner product in $\mathbb{R}^{n},\|\cdot\|$ the euclidean norm, $B$ the open unit ball, and $\bar{B}$ its closure. We will also denote the distance between $x$ and the set $S$ by $d_{S}(x)$.

\section{Definitions and statements of the Results}

In this paper we are concerned with control systems of the form

$$
\dot{x}(t)=f(x(t), u(t))
$$

where the state $x(t)$ is in the Euclidean space $\mathbb{R}^{n}$ and the control $u(t)$ belongs to the set of control $U$ which is assumed to be a subset of $\mathbb{R}^{m}$. Moreover we will assume that the function $f$ is continuous from $\mathbb{R}^{n} \times U$ into $\mathbb{R}^{n}$ and that it admits an equilibrium point at the origin, i.e. $f(0,0)=0$ (where " 0 " is some particular control in $U)$. We recall that the trajectories of $(2.1)$ are absolutely continuous functions $x(\cdot)$ associated with some open loops $u:[0, \infty[\rightarrow U$ and solutions of (2.1) almost everywhere.

Our objective is to find a necessary and sufficient condition for the existence of a smooth control-Lyapunov function. Let us give the definition of this concept. Note before that the function $V: \mathbb{R}^{n} \longmapsto \mathbb{R}_{\geq 0}$ is positive definite if $x \neq 0 \Rightarrow V(x)>0$ and $V(0)=0$ and that it is proper if $\lim _{\|x\| \rightarrow \infty} V(x)=\infty$.

Definition 2.1. Let $V$ be an application which is assumed to be $C^{1}$ from $\mathbb{R}^{n}$ into $\mathbb{R}$. It is said to be a controlLyapunov function (abreviated clf) for the system (2.1) if it is positive definite, proper and if the following condition is satisfied: for any compact set $K$ of $\mathbb{R}^{n}$, there exists a compact subset $U_{0} \subset U$ such that

$$
\forall x \in K \backslash\{0\}, \min _{u \in U_{0}}\langle\nabla V(x), f(x, u)\rangle<0 .
$$

As it has been shown by Kurzweil [20] (and by Massera [23] in the local case) in the context of classical differential equations, if the system $\dot{x}=g(x)$ is globally asymptotically stable at the origin then there exists a (classical) Lyapunov function. If the system (2.1) admits a continuous stabilizing feedback, that is, a continuous function

$$
u: \mathbb{R}^{n} \longrightarrow U
$$


such that the closed-loop system $\dot{x}=f(x, u(x))$ is globally asymptotically stable, then there exists a smooth $\left(C^{\infty}\right)$ control-Lyapunov function. Unfortunatly, the converse is false; the presence of a smooth control-Lyapunov function does not lead to the existence of continuous stabilizing feedbacks. Let us give the following example (quoted by Sontag in [30]) to highlight this problem:

$$
\begin{aligned}
& \dot{x}_{1}=u_{2} u_{3} \\
& \dot{x}_{2}=u_{1} u_{3} \\
& \dot{x}_{3}=u_{1} u_{2}
\end{aligned}
$$

where $\left(u_{1}, u_{2}, u_{3}\right) \in \bar{B}$ is the unit ball of $\mathbb{R}^{3}$.

The function $x \mapsto\|x\|^{2}$ is clearly a smooth clf for this system and yet it does not admit a continuous stabilizing feedback. As a matter of fact, no point of the form $(0, \epsilon, \epsilon)$ belongs to the set $f(x, u)$ for $x$ in a neighbourhood of the origin and $u$ in the unit ball $B$. In other words, the system (2.3) does not satisfy the Brockett's condition which is necessary for the existence of continuous feedback. This example leads us to consider another form of feedback. We will deal with differential inclusions that we proceed to define.

Let $F: \mathbb{R}^{n} \rightarrow 2^{\mathbb{R}^{n}}$ be a multivalued map. We shall say that the hypotheses $(\mathrm{H})$ are satisfied if the two following conditions hold:

(H1) For any $x$ in $\mathbb{R}^{n}, F(x)$ is a nonempty compact convex set.

(H2) The multivalued map $F$ is upper semicontinous, that is:

$\forall x \in \mathbb{R}^{n}, \forall \epsilon>0, \exists \delta>0$ such that

$$
\|x-y\|<\delta \Rightarrow F(y) \subset F(x)+\epsilon B
$$

The hypotheses $(\mathrm{H})$ correspond to the usual framework for the study of the differential inclusion

$$
\dot{x}(t) \in F(x(t)) \quad \text { a.e. }
$$

associated with $F$. For the sake of simplicity, we will sometimes speak about the multivalued map $F$ for talking about its differential inclusion (2.4). We suggest the following references for a detailed study of multivalued maps and differential inclusions $[4,13]$ and [3].

Knowing this notion we are now able to extend the classical definition of asymptotic stability to the more general case of differential inclusions.

Definition 2.2. The differential inclusion (2.4) is Globally Asymptotically Stable (abreviated GAS) provided that no solution exhibits finite time blow-up, and provided that the following holds:

(a) Uniform Attraction: for any $0<r<R$, there exists $T=T(r, R)>0$ such that for any solution $x(\cdot)$ of (2.4) with $\|x(0)\| \leq R$, one has

$$
\|x(t)\| \leq r \quad \forall t \geq T
$$

(b) Uniform Boundedness: there is a continuous nondecreasing function

$$
M:] 0, \infty[\rightarrow] 0, \infty[
$$

such that for any solution $x(\cdot)$ of $(2.4)$, one has

$$
\|x(t)\| \leq M(\|x(0)\|) \quad \forall t \geq 0 .
$$

(c) Lyapunov Stability:

$$
\lim _{R \downarrow 0} M(R)=0 .
$$

Remark 2.3. This definition agrees with the one taken in [8] and [22]. 
The concept of differential inclusion will allow us to give a sense to the Cauchy problem $\dot{x}(t)=f(x(t), u(x(t)))$ when the function $x \mapsto f(x, u(x))$ does not have continuity properties. As a matter of fact, in the general case of non necessarly continuous functions, no theorem insures the existence of solution to the Cauchy problem

$$
\dot{x}(t)=f(x(t), u(x(t))), \quad x(0)=x_{0} .
$$

That's why we have to recall an alternative concept of solutions.

Let be given

$$
u: \mathbb{R}^{n} \rightarrow U,
$$

an application which is not assumed to be continuous. We shall say that the function $u$ is locally bounded if, for any compact set $K$ of $\mathbb{R}^{n}$, there exists $\delta_{K}>0$ such that for any $x \in K$

$$
\forall \delta \leq \delta_{K}, \overline{u(x+\delta B)} \text { is a compact set in } \mathbb{R}^{m} .
$$

We can associate two multivalued maps with the function $u$ as follows. We set for any $x$ in $\mathbb{R}^{n}$ :

$$
\begin{gathered}
K_{u}(x):=\overline{\mathrm{co}}\left\{\cap_{\delta>0} f(x, u(x+\delta B))\right\}, \\
\text { and } \quad F_{u}(x):=\overline{\mathrm{co}}\left\{\bigcap_{\lambda(N)=0} \bigcap_{\delta>0} f(x, u(x+\delta B \backslash N))\right\},
\end{gathered}
$$

where co $A$ denotes the convex hull of the set $A$ and where $\lambda$ denotes the Lebesgue measure on $\mathbb{R}^{n}$.

The multivalued maps $K_{u}$ and $F_{u}$ possess nice properties of regularity. If $u$ is locally bounded, then $K_{u}$ satisfies hypotheses $(\mathrm{H})$ and if moreover $u$ is also measurable then $F_{u}$ satisfies $(\mathrm{H})$ too. Under these assumptions, we shall say that the absolutely continuous $\operatorname{arc} x(\cdot)$ is a solution of Krasovskii type (resp. of Filippov type) of the Cauchy problem (2.5) if it is solution of the differential inclusion (2.6) (resp. (2.7)). This leads to the following definition.

Definition 2.4. We shall say that the control system (2.1) admits a stabilizing feedback of Krasovskii type (resp. of Filippov type) if there exists a function $u: \mathbb{R}^{n} \longmapsto \mathbb{R}^{m}$ which is locally bounded and such that the differential inclusion (2.6) (resp. (2.7)) is Globally Asymptotically Stable.

We are going to connect the existence of a stabilizing feedback to the one of a control-Lyapunov function. As we said in the introduction, it is not necessary to work with smooth control-Lyapunov functions. Let us introduce some concepts of nonsmooth analysis.

Let $f: \mathbb{R}^{n} \longmapsto \mathbb{R}$ be a locally Lipschitz function, we denote by $\partial_{P} f(\bar{x})$ its proximal subdifferential at $\bar{x}$, i.e. the set of vectors $\zeta \in \mathbb{R}^{n}$ such that there exists $\delta, \sigma>0$ verifying

$$
f(y)-f(\bar{x})+\sigma\|y-\bar{x}\|^{2} \geq\langle\zeta, y-\bar{x}\rangle \quad \forall y \in \bar{x}+\delta B .
$$

This object permits us to define the generalized gradient of $f$ at $\bar{x}$ :

$$
\partial f(\bar{x}):=\operatorname{co}\left\{\lim \zeta_{k}: x_{k} \rightarrow \bar{x}, \zeta_{k} \in \partial_{P} f\left(x_{k}\right)\right\} .
$$

This tool has been introduced by Clarke in 1973. Since this time, a complete calculus has been developed, one that extends all the theorems of the usual smooth calculus. In particular, the generalized gradient of the sum of two functions is included in the sum of the generalized gradients. 
Remark 2.5. The definition of the generalized gradient given above coincides with the following one which is based on the Rademacher's theorem:

$$
\partial f(\bar{x}):=\operatorname{co}\left\{\lim \nabla f\left(x_{k}\right): x_{k} \rightarrow \bar{x}, x_{k} \in D_{f}\right\}
$$

where $D_{f}$ denotes the set of points where $f$ is differentiable.

We proceed now to adapt the classical defnition of control-Lyapunov function to the case of functions which are only locally Lipschitz.

Definition 2.6. Let $V: \mathbb{R}^{n} \longmapsto \mathbb{R}$; it is said to be a control-Lyapunov function in the sense of generalized gradients for the system (2.1) if $V$ is locally Lipschitz, positive definite, proper and if it satisfies the following property: for any compact set $K$ in $\mathbb{R}^{n}$, there exists $U_{0} \subset U$ such that

$$
\forall x \in K \backslash\{0\}, \forall \zeta \in \partial V(x), \min _{u \in U_{0}}\langle\zeta, f(x, u)\rangle<0 .
$$

Like the proximal subdifferential in the case of systems which are globally asymptotically controllable (see $[6,26])$, the generalized gradient constitutes a fantastic tool for the study of systems which possess strong properties of stability. Let us state our main result.

Theorem 2.7. The following properties are equivalent:

(i) the system (2.1) admits a control-Lyapunov function in the sense of generalized gradients;

(ii) the system (2.1) admits a control-Lyapunov function which is $C^{\infty}$ on $\mathbb{R}^{n}$;

(iii) the system (2.1) admits a stabilizing feedback of Krasovskii type (or of Filippov type).

The parts $($ iii $) \Rightarrow($ i $)$ and $($ iii $) \Rightarrow($ ii $)$ are not new. However, we present a proof which differs from the known ones (see Clarke et al. [8], Lin et al. [22] and more recently Praly and Teel [31]). Instead of constructing a global locally Lipschitz differential inclusion to deduce the existence of a control-Lyapunov function in the sense of generalized gradients, we design locally some control-Lyapunov functions that we glue to obtain a global clf. This approach requires some easy facts of the nonsmooth calculus and makes the proof inedite. Actually this kind of construction could be related to the one used in the proof of the existence of a locally Lipschitz control-Lyapunov function for asymptotically controllable systems, see [26]. On the other hand, we precise that the proof of $(i) \Rightarrow(i i)$ is inspired by ideas given in [8]. However, in our context, we deal with weak controlLyapunov functions (i.e. we have a minimum over $U$ instead of a maximum in the decrease property (2.8)) that require a more technical proof.

Furthermore, we find the Artstein's result (also given in [28] and [12]) in the case of $f$ affine in the control; that is, when the function $f$ is of the form

$$
f(x, u)=f_{0}(x)+\sum_{i=1}^{m} u_{i} f_{i}(x)
$$

where the $f_{i}$ 's are some locally Lipschitz functions from $\mathbb{R}^{n}$ into $\mathbb{R}^{n}$. In addition we are able to precise the regularity of the stabilizing feedback given in (iii) under an additional assumption around the origin. Let us state the result.

Theorem 2.8. If $f$ is affine in the control and if the property (i) of Theorem 2.7 holds (or equivalently (ii)) then there exists a stabilizing feedback which is continuous on $\mathbb{R}^{n} \backslash\{0\}$. If moreover for all $\epsilon>0, \exists \delta>0$ such that if $\|x\|<\delta$ then the compact set $W_{x}$ of Definition 2.1 can be taken to be included in $\epsilon B$, then the stabilizing feedback can be taken to be continuous at the origin.

We present now a nice example where our results apply. 


\section{Application to the integrator problem}

In order to highlight the using of nonsmooth clf, we present a result which concerns the system with integrator associated with the initial system (2.1). This kind of problem has been studied recently in [16,25] where we can recognize the nonsmooth method which will be used. Our proof is inspired by the one given by Byrnes and Isidori [5] and Tsinias [33] in the smooth case; one can also find this proof in the book of Sontag [29]. On the other hand, we stress that Rosier [27] provides an alternative demonstration of (3.1) in the smooth case; it does not involve control-Lyapunov function. Besides, we suggest more additional readings to the curious reader on other questions related to the integrator problem $[10,11]$.

Theorem 3.1. Let $f: \mathbb{R}^{n} \times U \rightarrow \mathbb{R}^{n}$ a function which is assumed to be continuous in $x$ and locally Lipschitz in $u$ uniformly in $x$ in a neighbourhood of the origin in $\mathbb{R}^{n} \times \mathbb{R}^{m}$. If the control system (2.1) possesses a locally Lipschitz stabilizing feedback, then the control system with integrator

$$
\left\{\begin{array}{l}
\dot{x}=f(x, u) \\
\dot{u}=v \in \mathbb{R}^{m}
\end{array}\right.
$$

admits a stabilizing feedback which is continuous on $\mathbb{R}^{n}$.

Proof. Let us denote $k$ the locally Lipschitz feedback which stabilizes our system. By Theorem 2.7, there exists a smooth control-Lyapunov function $V$ on $\mathbb{R}^{n}$ verifying in particular:

$$
\forall x \neq 0,\langle\nabla V(x), f(x, k(x))\rangle<0
$$

Consider the function $W$ from $\mathbb{R}^{n} \times \mathbb{R}^{m}$ into $\mathbb{R}$

$$
\forall(x, z) \in \mathbb{R}^{n} \times \mathbb{R}^{m}, W(x, z)=V(x)+\frac{1}{2}\|z-k(x)\|^{2} .
$$

We shall prove that the function $W$ is a control-Lyapunov function in the sense of generalized gradients. Therefore, we will conclude by Theorem 2.8 .

First of all, it is easy to see that $W$ is locally Lipschitz, positive definite and proper. Let us look at the generalized gradients of $W$.

Take $(\bar{x}, \bar{z}) \in \mathbb{R}^{n} \times \mathbb{R}^{m}$ and $\left(\zeta_{1}, \zeta_{2}\right) \in \partial W(\bar{x}, \bar{z})$ where $\zeta_{1} \in \mathbb{R}^{n}$ and $\zeta_{2} \in \mathbb{R}^{m}$.

We see immediatly that $\zeta_{2}=\bar{z}-k(\bar{x})$.

Moreover, by a classical chain rule on the generalized jacobians (see [7] or [18]), one has

$$
\zeta_{1}=\nabla V(\bar{x})+\zeta_{1}^{\prime}
$$

with $\left\|\zeta_{1}^{\prime}\right\| \leq L_{k}\|\bar{z}-k(\bar{x})\|$, where $L_{k}$ is the Lipschitz constant of $k$ in a neighbourghood of $\bar{x}$. We deduce that

$$
\partial W(\bar{x}, \bar{z}) \subset\left(\nabla V(\bar{x})+L_{k}\|\bar{z}-k(\bar{x})\| \bar{B}, \bar{z}-k(\bar{x})\right)
$$

Consider now $(x, z) \in \mathbb{R}^{n} \times \mathbb{R}^{m} \backslash(0,0)$ and $\left(\zeta_{1}, \zeta_{2}\right) \in \partial W(x, z)$. We can write $\left(\zeta_{1}, \zeta_{2}\right)=(\nabla V(x)+\Psi,(z-k(x)))$ with $\|\psi\| \leq L_{k}\|z-k(x)\|$.

First case: if $z=k(x)$, then $\left(\zeta_{1}, \zeta_{2}\right)=(\nabla V(x), 0)$ and the decrease condition (2.8) is satisfied by (3.2).

Second case: if $\|z-k(x)\|>0$.

We define $M$ a matrix of $M_{n, m}(R)$ as follows:

$$
M:=\frac{f(x, z)-f(x, k(x))(z-k(x))^{t}}{\|z-k(x)\|^{2}} .
$$


It is straightforward to show that $\|M\|$ is bounded by the Lipschitz constant of $f$ in the variable $z$ with $x$ fixed. We set:

$$
v:=k(x)-z-M^{t} \nabla V(x)+\mu \text { where } \mu:=\frac{k(x)-z}{\|z-k(x)\|^{2}}\langle\Psi, f(x, z)\rangle .
$$

We can compute by $(3.2)$ :

$$
\begin{aligned}
\left\langle\left(\zeta_{1}, \zeta_{2}\right),(f(x, z), v)\right\rangle & =-\|z-k(x)\|^{2}+\langle\nabla V(x), f(x, z)-M(z-k(x))\rangle \\
& =-\|z-k(x)\|^{2}+\langle\nabla V(x), f(x, k(x))\rangle<0
\end{aligned}
$$

Moreover, if $(x, z)$ is in a small enough neighbourhood $\mathcal{V} \times \mathcal{W}$ of the origin, then by the hypotheses the function $f$ is locally Lipschitz in the variable $u$ uniformly in $x$; that is there exists a constant $L_{f}^{0}$ such that

$$
\forall x \in \mathcal{V}, \forall\left(u_{1}, u_{2}\right) \in \mathcal{W} \times \mathcal{W},\left\|f\left(x, u_{1}\right)-f\left(x, u_{2}\right)\right\| \leq L_{f}^{0}\left\|u_{1}-u_{2}\right\|
$$

We deduce that the matrix $M$ that we defined above is such that $\|M\| \leq L_{f}^{0}$. If we denote by $L_{k}^{0}$ the Lipschitz constant of the function $k$ on $\mathcal{W}$, then we get:

$$
\|v\| \leq\|z-k(x)\|+L_{f}^{0}\|\nabla V(x)\|+L_{k}^{0}\|f(x, z)\|
$$

where $(x, z)$ belongs to $\mathcal{V} \times \mathcal{W}$ and $v$ corresponds to $v(=v(x, z))$ from above.

Hence we deduce that the quantity $v(=v(x, z))$ tends to 0 when the couple $(x, z)$ converge to the origin. This enables us to apply Theorem 2.8 and to obtain the existence of a stabilizing feedback $v(\cdot, \cdot)$ which is continuous on $\mathbb{R}^{n}$.

We will try now to understand why we cannot relax the hypotheses of Theorem 3.1 into continuity of the stabilizing feedback $k$. First of all, we know a counterexample in this kind of situation; Coron and Rosier provide in [12] the following control system:

$$
\dot{x}=f(x, u)=x^{\frac{1}{3}}-u
$$

(The function $f$ is continuous and Lipschitz in $u$ uniformly in $x$.)

The feedback law $u(x)=2 x^{\frac{1}{3}}$ is a stabilizing feedback and moreover there does not exist other stabilizing feedbacks which are locally Lipschitz at the origin. Let us now looking at the integrator system

$$
\begin{cases}\dot{x} & =x^{\frac{1}{3}}-u \\ \dot{u} & =v\end{cases}
$$

It does not possess any continuous stabilizing feedback. Coron and Rosier give the following function:

$$
V(x, u)=x-u^{2} .
$$

Along a trajectory $(x(t), u(t))$ of the integrator system, it satisfies:

$$
\dot{V}=x^{\frac{1}{3}}-u-2 u v \geq 0
$$

whenever $V$ is nonnegative, $v \leq 1$ and $|(x, u)|$ is sufficently small.

But if there would exist a stabilizing feedback continuous at the origin, then it would verify the Lyapunov Stability property (see Def. 2.2). But every point of the set $A:=\left\{(x, u): x-u^{2} \geq 0\right\}$ can not reach asymptotically the origin without leaving the part $A$ where $\dot{V}$ is positive. In other terms, in order to tends to 0 the stabilizing trajectories must move away from the origin. As we are seeing, the integrator system does not possess continuous stabilizing feedbacks because the Lyapunov stability property can not be satisfied. Actually, 
for $\left(x_{0}, u_{0}\right)$ close enough to the origin, there does not exist stabilizing trajectories which converge to zero with respect to small controls! The system is not stabilizable at the origin with small controls, how could it be stabilizable by a continuous feedback or even by a continuous "time-varying feedback" (see [12])?

In a general manner, if we assume that the initial control system (2.1) admits a stabilizing feedback which is only continuous at the origin, then it seems to be difficult to prove that the integrator system (3.1) is controllable at the origin with small controls. As a matter of fact, let be given an initial state $\left(x_{0}, u_{0}\right)$, if we consider a trajectory $x(\cdot)$ of the GAS system $\dot{x}=f(x, k(x))$ on $[0, \infty)$. It converges to the origin and moreover we can compute:

$$
\dot{u}(t)=d k(x(t)) \cdot f(x(t), k(x(t)) .
$$

Thus the control $v:=\dot{u}(t)$ drives the system (3.1) to the origin, but we cannot claim that the controls are sufficiently small around the origin (the term $d k(x(t)$ might be very big).

To conclude, if we omit the assumption of Lipschitz regularity on $k$, we cannot prove the existence of a stabilizing feedback (for the integrator system) which is continuous at the origin. However, it is in fact possible to get a stabilizing feedback which is continuous (even smooth) outside the origin. In other words, we can produce a control-Lyapunov function which is sufficiently regular $\left(C^{1}\right.$ or locally lipschitz) but which does not verify the small control property (see Artstein [1] or the last assumption of Th. 2.8). Such results have been proven by Tsinias; we refer to [34] for the case $m=1$ and to [35] for the multi-input case.

\section{Proofs Theorems 2.7 And 2.8}

Let us begin by the most difficult part: (iii) $\Longrightarrow$ (i).

In fact we will derive our result from the following theorem.

Theorem 4.1. Let $F: \mathbb{R}^{n} \rightarrow 2^{\mathbb{R}^{n}}$ be a multivalued function satisfying the hypotheses $(H)$ and such that the differential inclusion (2.4) is GAS. Then under these assumptions, there exists a function $V: \mathbb{R}^{n} \rightarrow \mathbb{R}$ which is locally Lipschitz, positive definite, proper and such that:

$$
\forall x \neq 0, \forall \zeta \in \partial V(x), \forall v \in F(x),\langle\zeta, v\rangle<0 .
$$

Remark 4.2. In this case, the application $V$ is called strong control-Lyapunov function for $F$. By the same proof as for (i) $\Rightarrow$ (ii), one can deduce from Theorem 4.1 the existence of a smooth strong control-Lyapunov function $F$; we recognize the main result given by Clarke et al. [8].

Let us consider $F$ a multivalued application being GAS; we are going to construct a strong control-Lyapunov function. Let us before change the dynamic to transform it into a bounded dynamic.

Lemma 4.3. Let $F$ be a multifunction which satisfies $(H)$, let $\alpha: \mathbb{R}^{n} \rightarrow[0, \infty[$ be a positive definite continuous function. Then the multifunction defined as follows

$$
F_{\alpha}(x):=\alpha(x) F(x)
$$

satisfies (H) and is GAS.

We suggest the reading of [8] for a proof of this result. Up to set $F(x):=F_{\alpha}(x)$ with $\alpha(x):=[\max \{1+\|v\|$ : $v \in F(x)\}]^{-1}$, we can assume that $F$ is bounded by 1 on $\mathbb{R}^{n}$. Besides, since $\alpha$ does not vanish outside the origin, a Lyapunov function for $F$ is obviously a control-Lyapunov function for $F_{\alpha}$.

The proof of our Theorem requires the following compactness property of solutions of differential inclusions (see e.g. $[3,7,9,13])$. 
Lemma 4.4. Let $F$ be a multivalued map satisfying hypotheses $(H)$. Then for any sequence $\delta_{k} \rightarrow 0$ and sequence $x_{k}(\cdot)$ of absolutely continuous and uniformly bounded functions on $[a, b]$ satisfying

$$
\dot{x_{k}}(t) \in \overline{\mathrm{co}} \quad F\left(x_{k}(t)+\delta_{k} \bar{B}\right)+\delta_{k} \bar{B} \text { a.e. }
$$

there exists a subsequence $x_{k_{i}}(\cdot)$ converging uniformly to some solution $x(\cdot)$ of $\dot{x} \in F(x)$ on $[a, b]$.

Give now the proposition which will be fundamental in the construction of the control-Lyapunov function in the sense of generalized gradients. We will invoke it many times with different functions $G$.

Proposition 4.5. Let $G$ be a multivalued map from $\mathbb{R}^{n}$ into $2^{\mathbb{R}^{n}}$ satisfying (H1) and which is locally Lipschitz and bounded (by a constant $m$ ), let $L: \mathbb{R}^{n} \rightarrow \mathbb{R}$ be a locally Lipschitz function and $[0, b]$ be an interval on the real line. Then the value function defined as follows

$$
V(x):=\max \left\{\int_{0}^{b} L(x(t)) \mathrm{d} t\right\}
$$

where the maximum is taken over all trajectories of

$$
\dot{x}(t) \in G(x(t)) \text { a.e. }
$$

with $x(0)=x$, is locally Lipschitz on $\mathbb{R}^{n}$; moreover it satisfies the following property

$$
\forall \zeta \in \partial V(x), \forall v \in G(x),\langle\zeta, v\rangle \leq-L(x)+L_{x, b}
$$

where $L_{x, b}$ denotes the maximum of $L(x(b))$ over all the trajectories of (4.2) starting at $x$ and defined on [0,b].

Remark 4.6. Since the multivalued map $G$ is uniformly bounded, the reachable sets of its corresponding differential inclusion at time $b$ are compact and hence the $L_{x, b}$ 's are well-defined.

Proof. Let us begin by proving that the value function $V$ is locally Lipschitz on $\mathbb{R}^{n}$; fix $r>0$. Consider $x$ in $r \bar{B}$. Since the multifunction $G$ is bounded by $m$, for any trajectory $z(\cdot)$ of $(4.2)$ with $z(0)=z$, one has $\|z(t)\| \leq\|z\|+t m, \forall t \in[0, b]$. If we consider a sequence of trajectories $\left(x_{n}(\cdot)\right)_{n}$ such that the quantities

$$
\int_{0}^{b} L\left(x_{n}(t)\right) \mathrm{d} t
$$

converge to $V(x)$, then all stay in the ball $(r+b m) \bar{B}$; this means that they are uniformly bounded. Hence we can apply the compactness of trajectories (see [9]) to deduce that the maximum in the definition of $V(x)$ is attained for some trajectory $\tilde{x}(\cdot)$ of $(4.2)$ with $\tilde{x}(0)=x$. Besides for any $y \in r \bar{B}$, by [3] (Cor. 1, p. 121) there exists a trajectory $y(\cdot)$ of $(4.2)$ with $y(0)=y$ which satisfies

$$
\forall t \in[0, b],\|y(t)-\tilde{x}(t)\| \leq \mathrm{e}^{\lambda_{G} b}\|y-x\|,
$$

where $\lambda_{G}$ is the Lipschitz constant of $G$ on $(r+m b) \bar{B}$. If we set $\lambda_{L}$ the Lipschitz constant of $L$ on the ball $(r+m b) \bar{B}$, we obtain

$$
V(x)=\int_{0}^{b} L(\tilde{x}(s)) \mathrm{d} s \leq \int_{0}^{b} L(y(s)) \mathrm{d} s+\int_{0}^{b} \lambda_{L}\|y(s)-\tilde{x}(s)\| \mathrm{d} s \leq V(y)+b \lambda_{L} \mathrm{e}^{\lambda_{G} b}\|y-x\| .
$$

Since we can repeat the same thing by inverting $x$ and $y$ and by varying $r$, we deduce that the function $V$ is locally Lipschitz on $\mathbb{R}^{n}$. We proceed now to prove inequality (4.3) for $x_{0}$ fixed in $\mathbb{R}^{n}$. Let us begin by 
verifying (4.3) with the elements of the proximal subdifferential. Let $\zeta \in \partial_{P} V\left(x_{0}\right)$ and $v \in G\left(x_{0}\right)$. By the famous Michael's Theorem [24], there exists a continuous map (we can even get it locally Lipschitz, see [4]) $g: \mathbb{R}^{n} \rightarrow \mathbb{R}^{n}$ such that $\forall x \in \mathbb{R}^{n}, g(x) \in G(x)$ and $g\left(x_{0}\right)=v$. Consider $x(\cdot)$ a solution of the classical ordinary differential equation $\dot{x}=g(x(t))$ on $[0, b]$ with $x(0)=x_{0}$, it is a trajectory of (4.2). Thus we obtain by definition of $V$, for any $t \in[0, b]$ :

$$
V(x(t))+\int_{0}^{t} L(x(s)) \mathrm{d} s-\int_{b}^{b+t} L\left(\tilde{x}^{t}(s-t)\right) \mathrm{d} s \leq V\left(x_{0}\right)
$$

where $\tilde{x}^{t}(\cdot)$ denotes a trajectory which realizes the maximum in the definition of $V(x(t))$, that is such that

$$
V(x(t))=\int_{0}^{b} L\left(\tilde{x}^{t}(s)\right) \mathrm{d} s=\int_{t}^{b+t} L\left(\tilde{x}^{t}(s-t)\right) \mathrm{d} s .
$$

On the other hand, there exist $\sigma, \eta>0$ such that

$$
V(y)-V\left(x_{0}\right)+\sigma\left\|y-x_{0}\right\|^{2} \geq\left\langle\zeta, y-x_{0}\right\rangle \quad \forall y \in x_{0}+\eta B .
$$

Therefore by setting $y=x(t)$, we get for $t$ sufficently small:

$$
\left\langle\zeta, x(t)-x_{0}\right\rangle \leq \sigma\left\|x(t)-x_{0}\right\|^{2}+\int_{b}^{b+t} L\left(\tilde{x}^{t}(s-t)\right) \mathrm{d} s-\int_{0}^{t} L(x(s)) \mathrm{d} s .
$$

By dividing by $t$, and passing to the limit we obtain easily

$$
\langle\zeta, v\rangle \leq L_{x, b}-L\left(x_{0}\right) .
$$

This proves our inequality in the case of proximal subgradients. To conclude, we claim that the inequality (4.3) will remain by passing to the limit and then will be satisfied for the elements of the limiting subdifferential $\partial_{L} V\left(x_{0}\right)$. The inequality will also remain by passing to the convex hull. The proposition is proved.

Come back to our proof; set new things from the multivalued function $F$. Let be given a positive number $\delta$, we shall call $F_{\delta}$ the multifunction defined as follows: for any $x \in \mathbb{R}^{n}$,

$$
\dot{x}(t) \in F_{\delta}(x(t)):=\overline{\mathrm{CO}} \quad F(x(t)+\delta \bar{B})+\delta \bar{B} .
$$

We proceed now to present a lemma which can be found in [8]; we give its proof for being more self-contained.

Lemma 4.7. Let $R, T, \epsilon$ be given positive numbers. Then there exists $\Delta=\Delta(R, T, \epsilon)$ such that the following hold: for any $\delta \in[0, \Delta]$, each solution $x(\cdot)$ of (4.4) with $\|x(0)\| \leq R$ does not blow-up on $[0, T]$ and satisfies

(a) $\|x(t)\| \leq 2 M(R) \quad \forall t \in[0, T]$.

(b) There exists a solution $\tilde{x}(\cdot)$ of (2.4) on $[0, T]$ such that

$$
\|x(t)-\tilde{x}(t)\| \leq \epsilon \quad \forall t \in[0, T] .
$$

Proof. If there exist sequences $\delta_{i} \downarrow 0$ and $T_{i}$ and corresponding solutions $x_{i}(\cdot)$ of $(4.4)$ on $\left[0, T_{i}\right]$ with $\delta=\delta_{i}$ and $\left\|x_{i}(0)\right\| \leq R$, such that

$$
\left\|x_{i}\left(T_{i}\right)\right\|=2 M(R),\left\|x_{i}(t)\right\|<2 M(R) \quad \forall t \in\left[0, T_{i}[.\right.
$$

Since the multifunction $F$ is bounded on the ball $2 M(R) B$, then all the $F_{\delta_{i}}$ are bounded too, and even uniformly. Because of fact that $\left\|x_{i}\left(T_{i}\right)\right\|=2 M(R)$, this implies that the sequence $T_{i}$ does not admit 0 as a cluster point. Hence, we can assume that $T_{i} \uparrow T^{\prime} \leq T$. Thus, we can apply Lemma 4.4 to deduce the existence of a solution 
$x(\cdot)$ of $(2.4)$ which is a uniform limit of the $x_{i}$ 's on $\left[0, T^{\prime}\right]$. Consequently, it satisfies $\left\|x\left(T^{\prime}\right)\right\|=2 M(R)$. That brings a contradiction to the definition of $M(R)$ ! Therefore we have proved the existence of some $\Delta>0$ verifying the first part of the lemma. On the other hand, we have shown that any trajectory starting in the ball $R \bar{B}$ remains in $2 M(R) \bar{B}$ (whenever $t \leq T)$. Then we can always extend it to $[0, T]$.

Assume now that there exists a sequence $\delta_{i} \downarrow 0$ and corresponding trajectories $x_{i}(\cdot)$ of $(4.4)$ on $[0, T]$ with $\delta=\delta_{i}$ and $\left\|x_{i}(0)\right\| \leq R$, such that

$$
\max _{t \in[0, T]}\left\|x_{i}(t)-\tilde{x}(t)\right\| \geq \epsilon
$$

for any trajectory $\tilde{x}(\cdot)$ of $(2.4)$. The sequence being uniformly bounded (by the first part of the proof), Lemma 4.4 brings a contradiction. Finally, Lemma 4.7 is proved.

We are now able to prove the following:

Lemma 4.8. Let $0<r<R$ be two positive numbers, then there exist $\tilde{\Delta}=\tilde{\Delta}(r, R)>0$ and $\tilde{T}>0$ which satisfy:

For any $\delta \in[0, \Delta]$, any $x(\cdot)$ solution of (4.4) with $\|x(0)\| \leq R$ can be extended to $[0, \infty)$ such that

$$
\begin{aligned}
& \|x(t)\| \leq 2 M(R) \quad \forall t \geq 0, \\
& \text { and } \quad\|x(t)\| \leq r \quad \forall t \geq \tilde{T} .
\end{aligned}
$$

Proof. By Lyapunov stability, we know that $M(\epsilon) \downarrow 0$ when $\epsilon \downarrow 0$, then there exists $0<\epsilon<r$ such that $2 M(\epsilon)<r$. By applying Lemma 4.7 with $T=T\left(\frac{\epsilon}{2}, R\right), \frac{\epsilon}{2}$ and $R$, we get the existence of $\Delta$ such that for any $\delta \in[0, \Delta]$, for any solution $x(\cdot)$ of $(4.4)$ on $[0, T]$, one has

$$
\|x(t)\| \leq 2 M(R) \quad \forall t \in[0, T]
$$

and there exists a solution $\tilde{x}(\cdot)$ of $(2.4)$ such that

$$
\|x(t)-\tilde{x}(t)\| \leq \frac{\epsilon}{2} \quad \forall t \in[0, T] .
$$

By applying still once Lemma 4.7 with $T=T\left(\frac{\epsilon}{2}, R\right)$ and $R=\epsilon$, we get $\Delta^{\prime}$ such that for any $\delta \in\left[0, \Delta^{\prime}\right]$, any solution $x(\cdot)$ of $(4.4)$ can be extended to $[0, T]$ and satisfies

$$
\| x(t \| \leq 2 M(\epsilon)<r \quad \forall t \in[0, T] .
$$

Set $\tilde{\Delta}:=\min \left\{\Delta, \Delta^{\prime}\right\}$. Let us prove that the properties (4.6) and (4.7) are verified for any $\delta$ in $[0, \tilde{\Delta}]$.

Let us consider such a $\delta$ and a solution $x(\cdot)$ of (4.4). It remains in the ball $2 M(R)$ whenever $t \leq T$, thus we will be able to extend it to $[0, T]$. Moreover, by construction of $\Delta$, there exists a trajectory $\tilde{x}(\cdot)$ of $(2.4)$ such that

$$
\|x(t)-\tilde{x}(t)\| \leq \frac{\epsilon}{2} \quad \forall t \in[0, T] .
$$

On the other hand, by definition of $T$, one has $\|\tilde{x}(T)\| \leq \frac{\epsilon}{2}$. Thus $\|x(T)\| \leq \epsilon \leq R$. Therefore, we can extend our trajectory on $[T, 2 T]$. Since $\|x(T)\| \leq \epsilon$, it remains in $2 M(\epsilon) \bar{B}$ and hence in $r \bar{B}$. As before $\|x(2 T)\| \leq \epsilon$. The work done above means that we can repeat the extension of our trajectory on the different intervals $[n T,(n+1) T]$ in such a manner that we stay in the ball $2 M(R) \bar{B}$ for $t \geq 0$ and in $r \bar{B}$ for $t \geq \tilde{T}=T\left(\frac{\epsilon}{2}, R\right)$. The lemma is proved.

We proceed now to construct our control-Lyapunov function in the sense of generalized gradients. For that, we start by setting a sequence of value functions $\left\{V_{p}\right\}_{p \geq 1}$ corresponding to the one that appeared in Proposition 4.5. 
Set for any positive integer $p, R_{p}:=2^{p}$. By Lemma 4.8, we can associate with each couple $\left(1,2 M\left(R_{p}\right)+2\right)$ some constants $\tilde{\Delta}_{p}$ and $\tilde{T}_{p}$ such that

$$
\tilde{\Delta}_{p+1} \leq \tilde{\Delta}_{p} \leq \tilde{\Delta}\left(1, R_{p}\right) \text { and } \tilde{T}_{p+1} \geq \tilde{T}_{p} \geq T\left(1,2 M\left(R_{p}\right)+2\right) .
$$

Fix now some positive integer $p$. We define a multifunction $F_{p}$, and then a new dynamics as follows.

Set for any $x$ in $\mathbb{R}^{n}$ :

$$
W_{x}^{p}:=\left\{y:\|y-x\| \leq \frac{1}{2} \tilde{\Delta}_{p}\right\} .
$$

Since the family $\left\{W_{x}^{p}\right\}_{x}$ is a covering of $\mathbb{R}^{n}$, there exists a locally finite open cover $\left\{W_{x_{i}}^{n}\right\}_{i}$ of $\mathbb{R}^{n}$ and $\left\{\Psi_{i}\right\}_{i}$ a subordinated $C^{\infty}$ partition of unity. We set for any $x$ in $\mathbb{R}^{n}$ :

$$
F_{p}(x):=\sum_{i} \Psi_{i}(x) F\left(x_{i}+\frac{\tilde{\Delta}_{p}}{2} \bar{B}\right) .
$$

By construction, $F_{p}$ satisfies obviously (H1), is locally Lipschitz and its norm is bounded by 1 on $\mathbb{R}^{n}$; on the other hand we have that for any $x$ in $\mathbb{R}^{n}$ :

$$
\begin{aligned}
F(x) \subset F_{p}(x) \subset \overline{\mathrm{CO}} F\left(x+\tilde{\Delta}_{p} \bar{B}\right) \subset F_{\tilde{\Delta}_{p}}(x), & \\
& \text { and } \\
F_{p+1}(x) & \subset \quad F_{p}(x) .
\end{aligned}
$$

Let us go back to the construction of our sequence of value functions. We shall apply Proposition 4.5 with each of the dynamics previously defined. We proceed recursively; let us start by defining $V_{1}$. We set for any $x$ in $\mathbb{R}^{n}$ :

$$
V_{1}(x):=\max \left\{\int_{0}^{\tilde{T}_{1}} L_{1}(x(s)) \mathrm{d} s: \dot{x} \in F_{1}(x(t)) \text { a.e. and } x(0)=x\right\},
$$

where $L_{1}(y)=d_{\bar{B}}(y) \quad \forall y \in \mathbb{R}^{n}$.

By Proposition 4.5, the value function $V_{1}$ is locally Lipschitz on $\mathbb{R}^{n}$ and proper (because the dynamics is globally bounded); moreover, it is nonnegative. Besides, if we consider $x \in \mathbb{R}^{n}$ with $\|x\| \leq 2 M\left(R_{1}\right)+2$, then by Lemma 4.8 for every trajectory $x(\cdot)$ of $(4.4)$ with $\delta=\tilde{\Delta}_{1}$ and $x(0)=x$, one has

$$
\|x(t)\| \leq 1 \quad \forall t \geq \tilde{T}_{1},
$$

that is $d_{\bar{B}}(x(t))=0$. The property (4.3) allows us to write for any $x \in \mathbb{R}^{n}$ with $\|x\| \leq 2 M\left(R_{1}\right)+2$ :

$$
\forall \zeta \in \partial V_{1}(x), \forall v \in F(x),\langle\zeta, v\rangle \leq-d_{\bar{B}}(x) .
$$

Let us now assume that we have defined the functions $V_{1}, V_{2}, \cdots, V_{p}$, and let us define the function $V_{p+1}$. We begin by setting two auxiliary functions; we set for any $x \in \mathbb{R}^{n}$ :

$$
B_{p}(x):=\max \left\{V_{p}(y):\|y\| \leq\|x\|+1\right\}+1,
$$

and

$$
L_{p+1}:=\max \left\{\frac{1}{2},\|x\|-2 M\left(R_{p}\right)\right\} L_{p}+\max \left\{0,\|x\|-2 M\left(R_{p}\right)\right\} B_{p}(x) .
$$


Define our function $V_{p+1}$; set for any $x$ in $\mathbb{R}^{n}$ :

$$
V_{p+1}(x):=\max \left\{\int_{0}^{\tilde{T}_{p+1}} L_{p+1}(x(s)) \mathrm{d} s: \dot{x} \in F_{p+1}(x(t)) \text { a.e. and } x(0)=x\right\} .
$$

Since the functions $B_{p}$ and $L_{p+1}$ are locally Lipschitz and nonnegative (positive outside $\bar{B}$ ), and since

$$
\tilde{T}_{p+1} \geq T\left(1,2 M\left(R_{p+1}\right)+2\right)
$$

Proposition 4.5 implies that $V_{p+1}$ is locally Lipschitz, proper, nonnegative (positive outside $\bar{B}$ ) and satisfies for any $x$ with $\|x\| \leq 2 M\left(R_{p+1}\right)+2$ :

$$
\forall \zeta \in \partial V_{p+1}(x), \forall v \in F(x),\langle\zeta, v\rangle \leq-L_{p+1}(x) .
$$

Furthermore, we have the following lemma:

Lemma 4.9. Let $x \in \mathbb{R}^{n}$.

(a) If $\|x\| \leq R_{p}$, then $V_{p+1}(x) \leq \frac{V_{p}(x)}{2}$.

(b) If $\|x\| \geq 2 M\left(R_{p}\right)+2$, then $V_{p+1}(x) \geq V_{p}(x)+1$.

Proof.

(a) Let $x \in R_{p} \bar{B}$. By Lemma 4.8 (because $\tilde{\Delta}_{p+1} \leq \tilde{\Delta}_{p} \leq \tilde{\Delta}\left(1, R_{p}\right)$ ) and from (4.8) applied for $p+1$, all the trajectories of $\dot{x} \in F_{p+1}(x)$ starting at $x$ stay in the ball $2 M\left(R_{p}\right) \bar{B}$. Thus, all along those we have:

$$
L_{p+1} \geq \frac{L_{p}}{2}
$$

In addition to that, by (4.9), these ones are also some trajectories of $\dot{x} \in F_{p}(x)$, and for all $t \geq \tilde{T}_{p}$, they remain in the unit ball, where $L_{p}$ vanishes. Since $\tilde{T}_{p+1} \geq \tilde{T}_{p}$, this implies that

$$
\int_{\tilde{T}_{p}}^{\tilde{T}_{p+1}} L_{p+1}(x(s)) \mathrm{d} s=0
$$

We conclude that

$$
V_{p+1}(x) \leq \frac{V_{p}(x)}{2}
$$

(b) Consider $x \notin\left[2 M\left(R_{p}\right)+2\right] B$. Since the dynamics $F_{p+1}$ has a norm bounded by 1 , all its trajectories $x(\cdot)$ starting at $x$ stay outside the ball $\left[2 M\left(R_{p}\right)+1\right] \bar{B}$ for $t \in[0,1]$. But from the definitions of $L_{p+1}$ and $B_{p}$, we have for any $t \in[0,1]$ :

$$
L_{p+1}(x(t)) \geq B_{p}(x(t)) \geq V_{p}(x)+1 .
$$

Hence we deduce that $V_{p+1}(x) \geq \int_{0}^{1} L_{p+1}(x(s)) \mathrm{d} s \geq V_{p}(x)+1$.

Since the construction of our sequence $\left(V_{p}\right)_{p \geq 1}$ is achieved, we can define:

$$
\forall x \in \mathbb{R}^{n}, \mathcal{V}_{0}(x):=\max \left\{V_{p}(x): p \geq 1\right\}
$$

This new function is well defined. As a matter of fact, the preceding lemma implies that if for some $q$ and $s$, we have $2 M\left(R_{q}\right)+2 \leq\|x\| \leq R_{s}$, then $\mathcal{V}_{0}(x)=\max _{q \leq p \leq s} V_{p}(x)$ and if $\|x\| \leq 2=R_{1}$, then $\mathcal{V}_{0}(x)=V_{1}(x)$. We conclude that the maximum in the definition of $\mathcal{V}_{0}(x)$ is attained only for a finite number of $V_{p}(x)$ 's. Moreover, since the functions $V_{p}$ are locally Lipschitz and proper, the function $\mathcal{V}_{0}$ is itself locally Lipschitz and proper. 
On the other hand, it is obviously nonnegative and positive outside the closed unit ball $\bar{B}$. In addition, by Proposition 2.3.12 of [7], if $\zeta$ belongs to $\partial \mathcal{V}_{0}(x)$ then it belongs to the convex hull of the $\partial V_{p}(x)$ for which the maximum in (4.12) is attained, that is such that $V_{p}(x)=\mathcal{V}_{0}(x)$. From these results and by (4.10) and (4.11) we deduce that:

$$
\forall x \in \mathbb{R}^{n} \backslash \bar{B}, \forall \zeta \in \partial \mathcal{V}_{0}(x), \forall v \in F(x),\langle\zeta, v\rangle<0
$$

On the other hand, it is easy to show that

$$
\forall x \in \bar{B}, \forall \zeta \in \partial \mathcal{V}_{0}(x), \forall v \in F(x),\langle\zeta, v\rangle \leq 0
$$

We set now a new sequence $r_{p}:=\frac{1}{2^{p}}$. As before, we can choose a sequence of couples $\left(\tilde{\Delta}_{p}^{\prime}, \tilde{T}_{p}^{\prime}\right)$ in such a manner that

$$
\tilde{T}_{p}^{\prime} \geq T\left(r_{p}, 2\right)
$$

We set as before a sequence $F_{p}^{\prime}$ of dynamics. Then we can set for any $p$ :

$$
V_{p}^{\prime}(x):=\max \left\{\int_{0}^{\tilde{T}_{p}^{\prime}} \mathrm{d}_{S_{p}}(x(s)) \mathrm{d} s: \dot{x} \in F_{p}^{\prime}(x(t)) \text { a.e. } \text { and } x(0)=x\right\}
$$

where $S_{p}$ denotes the ball $r_{p} \bar{B}$.

Each of these value functions $V_{p}^{\prime}$ is locally Lipschitz and satisfies by the good choice of $\tilde{T}_{p}^{\prime}$ :

$$
\forall x \in 2 \bar{B}, \forall \zeta \in \partial V_{p}^{\prime}(x), \forall v \in F(x),\langle\zeta, v\rangle \leq-d_{S_{p}}(x)
$$

By setting $M_{p}$ the maximum of $V_{p}^{\prime}$ over the ball $2 \bar{B}$, we can truncate it as follows:

We define for any $x \in \mathbb{R}^{n}$,

$$
\mathcal{V}_{p}:=\min \left\{M_{p}, V_{p}^{\prime}(x)\right\}
$$

First we mention that the maximum $M_{p}$ of $V_{p}^{\prime}$ is achieved on the boundary of $2 \bar{B}$ (this comes from (4.15) and the fact that 0 is a generalized gradient at the local maximum if it is in $2 B$ ). Furthermore Let us notice that $\mathcal{V}_{p}$ is globally Lipschitz (let us denote its Lipschitz constant by $K_{p}$ ) and bounded by $M_{p}$. There is in fact a connection between its generalized gradients and those $V_{p}^{\prime}$ :

$$
\forall x \in \bar{B}, \partial \mathcal{V}_{p}(x)=\partial V_{p}^{\prime}(x) \text { and } \forall x \notin \bar{B}, \partial \mathcal{V}_{p}(x) \subset \operatorname{co}\left\{0, \partial V_{p}^{\prime}(x)\right\}
$$

Finally, we set:

$$
\mathcal{V}(x):=\mathcal{V}_{0}(x)+\sum_{p=1}^{\infty} \rho_{p} \mathcal{V}_{p}(x),
$$

where $\rho_{p}:=\min \left\{\frac{1}{M_{p} 2^{p}}, \frac{1}{K_{p} 2^{p}}\right\}$. Still by construction, the function $\mathcal{V}$ is positive definite, proper and locally Lipschitz. Besides by a rule on the sum of generalized gradients (see [9]), we have:

If $\zeta \in \partial \mathcal{V}(x)$ then

$$
\zeta \in \partial \mathcal{V}_{0}(x)+\sum_{p=1}^{\infty} \rho_{p} \partial V_{p}(x)
$$

Hence by linearity of the scalar product, we get by (4.13-4.15) and (4.16):

$$
\forall x \neq 0, \forall \zeta \in \partial \mathcal{V}(x), \forall v \in F(x),\langle\zeta, v\rangle<0 .
$$

Theorem 4.1 is proved. 
We proceed now to show now the part (i) $\Longrightarrow$ (ii):

We assume that the system (2.1) admits a control-Lyapunov function in the sense of generalized gradients $V$; we shall regularize it.

Fix $x$ in $\mathbb{R}^{n} \backslash\{0\}$. By Definition 2.6, there exists a compact set $W_{x}$ such that

$$
\forall y \in 2\|x\| \bar{B} \backslash\{0\}, \forall \zeta \in \partial V(y), \min _{u \in W_{x}}\langle\zeta, f(y, u)\rangle<0
$$

We can define the following positive quantity:

$$
\epsilon_{x}:=-\max _{y \in x+\frac{\|x\|}{2}} \max _{\zeta \in \partial V(y)} \min _{u \in W_{x}}\langle\zeta, f(y, u)\rangle>0 .
$$

Note that the positivity of the constant $\epsilon_{x}$ is a consequence of the upper semicontinuity of the multivalued map $y \mapsto \partial V(y)$ and of the compacity of $W_{x}$.

We set also for any $y \in x+\frac{\|x\|}{2} \bar{B}$ :

$$
F_{x}(y):=\operatorname{co}\left\{f(y, u): u \in W_{x}\right\}
$$

Since $F_{x}(y)$ and $\partial V(y)$ are convex sets, the minimax Theorem (see [2]) implies by (4.17) and (4.18) that for any $y \in x+\frac{\|x\|}{2} \bar{B}$, there exists $v \in F_{x}(y)$ such that

$$
\forall \zeta \in \partial V(y),\langle\zeta, v\rangle \leq-\epsilon_{x}
$$

Furthermore, since the multivalued map $x \mapsto \partial V(x)$ is upper semicontinuous and since $F_{x}$ is continuous, we can find a neighbourhood $U_{x}$ (with $0 \notin \overline{U_{x}}$ ) of $x$ and a positive real number $\delta_{x}$ such that for any $y \in U_{x}$, there exists $v \in F_{x}(y)$ satisfying:

$$
\forall z \in U_{x}+\delta_{x} B, \forall \zeta \in \partial V(z),\langle\zeta, v\rangle \leq-\frac{\epsilon_{x}}{2}
$$

This construction being done for any $x$ in $\mathbb{R}^{n} \backslash\{0\}$, we can extract from the family $\left\{U_{x}\right\}_{x}$ a locally finite open cover $\left\{U_{i}\right\}_{i=1}^{\infty}$ of $\mathbb{R}^{n} \backslash\{0\}$ (where the $U_{i}$ 's are relatively compact open sets whom the closure does not contain the origin) corresponding to families $\left\{x_{i}\right\}_{i=1}^{\infty}$ and $\left\{\delta_{i}\right\}_{i=1}^{\infty}$. We set for any $x$ in $\mathbb{R}^{n} \backslash\{0\}$ :

$$
\delta(x):=\min \left\{\frac{\|x\|}{2}, \min \left\{\delta_{j}:\left(x+\frac{\|x\|}{2} \bar{B}\right) \cap U_{j} \neq \emptyset\right\}\right\}>0 .
$$

The family $\left\{x+\frac{\delta(x)}{2} B\right\}_{x}$ is an open cover of $\mathbb{R}^{n} \backslash\{0\}$; we can extract from it a locally finite open cover $\left\{x^{p}+\frac{\delta\left(x^{p}\right)}{2} B\right\}_{p=1}^{\infty}$ corresponding to a $C^{\infty}$ partition of unity $\{\psi\}_{p=1}^{\infty}$. For any $p, \operatorname{Supp}\left(\psi_{p}\right)$ will denote the support of the function $\psi_{p}$; let us recall that it is included in $x^{p}+\frac{\delta\left(x^{p}\right)}{2} B$. With this stuff, we can set for any $p$ :

$$
\begin{aligned}
\epsilon^{p} & :=\min _{\left(x^{p}+\frac{\left\|x^{p}\right\|}{2} \bar{B}\right) \cap U_{i} \neq \emptyset}\left\{\epsilon_{x_{i}}\right\}>0, \\
\bar{\epsilon}^{p} & :=\min \left\{\min _{\left.\left(x^{p}+\frac{\left\|x^{p}\right\|}{2} \bar{B}\right) \cap U_{i} \neq \emptyset \min _{x \in \bar{U}_{i}} V(x), \epsilon_{p}\right\}>0,} \max _{q_{p}}:=\max _{\left(x^{p}+\frac{\left\|x^{p}\right\|}{2} \bar{B}\right) \cap U_{i} \neq \emptyset x \in \bar{U}_{i}}\left\{\left\|\nabla W_{x_{i}}\right\|\|f(x, u)\|\right\}>0 .\right.
\end{aligned}
$$


Let $\omega: \mathbb{R}^{n} \rightarrow\left[0, \infty\left[\right.\right.$ be a $C^{\infty}$ function with support in the closed unit ball $\bar{B}$, such that

$$
\int_{\mathbb{R}^{n}} \omega(x) \mathrm{d} x=1 .
$$

For $\nu>0$ let us define for any $x \in \mathbb{R}^{n}$ :

$$
V_{\nu}(x):=\int_{\mathbb{R}^{n}} V(x+\nu y) \omega(y) \mathrm{d} y .
$$

This function is $C^{\infty}$ and furthermore if $S \subset \mathbb{R}^{n}$ is compact, then the sequence $\left(V_{\nu}\right)_{\nu}$ converges uniformly to $V$ when $\nu$ tends to 0 . Thus for each $p$, there exists $\nu_{p}<\frac{\delta\left(x^{p}\right)}{2}$ such that

$$
\left|V_{\nu_{p}}(x)-V(x)\right| \leq \frac{\bar{\epsilon}^{p}}{2^{p+1}\left(1+q_{p}\right)} \quad \forall x \in x^{p}+\delta\left(x^{p}\right) \bar{B} .
$$

Let us define a function

$$
\tilde{V}(x):= \begin{cases}\sum_{p} \psi_{p}(x) V_{\nu_{p}}(x) & \text { if } x \neq 0 \\ 0 & \text { if } x=0 .\end{cases}
$$

It is clearly differentiable on $\mathbb{R}^{n} \backslash\{0\}$. Furthermore, since for any $x \in \mathbb{R}^{n}$

$$
|\tilde{V}(x)-V(x)| \leq \sum_{p} \psi_{p}(x)\left|V(x)-V_{\nu_{p}}(x)\right| \leq \frac{1}{2} V(x)
$$

we obtain that the function $\tilde{V}$ is smooth outside the origin, positive definite, proper and continuous at the origin. Prove that $\tilde{V}$ satisfies the decrease condition (2.2).

From Rademacher's Theorem (see [9]), since $V$ is locally Lipschitz, it is differentiable almost everywhere in $\mathbb{R}^{n}$, and by Remark 2.5 , for every point $x$ where it is differentiable, we have

$$
\nabla V(x) \subset \partial V(x) .
$$

On the other hand, Lebesgue's theorem (because the function is locally Lipschitz) implies that for any $x \in$ $\mathbb{R}^{n} \backslash\{0\}$,

$$
\nabla V_{\nu}(x)=\int_{\mathbb{R}^{n}} \nabla V(x+\nu y) \omega(y) \mathrm{d} y .
$$

Fix $x \in \mathbb{R}^{n} \backslash\{0\}$. By construction of the family $\left\{U_{i}\right\}_{i=1}^{\infty}$, there exists $i_{0}$ such that $x \in U_{i_{0}}$. So if $x \in \operatorname{Supp}\left(\psi_{p}\right)$ for some $p$, then $x \in x^{p}+\frac{\delta\left(x^{p}\right)}{2} B$ and then $x^{p}+\frac{\delta\left(x^{p}\right)}{2} B \cap U_{i_{0}} \neq \emptyset$ that implies (since $\left.\delta\left(x^{p}\right) \leq\left\|x^{p}\right\|\right)$ :

$$
x^{p}+\frac{\left\|x^{p}\right\|}{2} B \cap U_{i_{0}} \neq \emptyset .
$$

We deduce by the definition of $\delta\left(x^{p}\right)$ that $\delta\left(x^{p}\right) \leq \delta_{i_{0}}$ and hence that $x+\frac{\delta\left(x^{p}\right)}{2} B$ is included in $U_{i_{0}}+\delta_{i_{0}} B$. On the other hand, there exists $v_{i_{0}} \in F_{x_{i_{0}}}(x)$ which satisfies (4.20) with $x=x_{i_{0}}$. We obtain by (4.25) and (4.26) that for any $p$ with $x \in \operatorname{Supp}\left(\psi_{p}\right)$ :

$$
\left\langle\nabla V_{\nu_{p}}(x), v_{i_{0}}\right\rangle=\int_{\mathbb{R}^{n}}\left\langle\nabla V\left(x+\nu_{p} y\right), v_{i_{0}}\right\rangle \omega(y) \mathrm{d} y \leq \int_{\mathbb{R}^{n}}-\epsilon_{x_{i_{0}}} \omega(y) \mathrm{d} y \leq-\epsilon_{x_{i_{0}}} .
$$

Now, if the function $V$ is differentiable at $x$, we can compute: 


$$
\begin{array}{ll} 
& \left\langle\nabla \tilde{V}(x), v_{i_{0}}\right\rangle \\
=\quad & \left\langle\nabla V(x), v_{i_{0}}\right\rangle+\sum_{p} \psi_{p}(x)\left\langle\nabla V_{\nu_{p}}(x)-\nabla V(x), v_{i_{0}}\right\rangle \\
& +\sum_{p}\left\langle\nabla \psi_{p}(x), v_{i_{0}}\right\rangle\left(V_{\nu_{p}}(x)-V(x)\right) \\
= & \sum_{p} \psi_{p}(x)\left\langle\nabla V_{\nu_{p}}(x), v_{i_{0}}\right\rangle+\sum_{p}\left\langle\nabla \psi_{p}(x), v_{i_{0}}\right\rangle\left(V_{\nu_{p}}(x)-V(x)\right) \\
\leq \quad & \sum_{p} \psi_{p}(x)\left(-\epsilon_{x_{i_{0}}}\right)+\sum_{p}\left\|\nabla \psi_{p}(x)\right\|\left\|v_{i_{0}}\right\|\left|V_{\nu_{p}}(x)-V(x)\right| \\
\leq \quad-\epsilon_{x_{i_{0}}}+\sum_{p} \frac{\bar{\epsilon}^{p}}{2^{p+1}} \\
\leq \quad-\frac{\epsilon_{x_{i_{0}}}}{2}<0 .
\end{array}
$$

Therefore since $v_{i_{0}}$ belongs to the convex hull of $\left\{f(x, u): u \in W_{x_{i_{0}}}\right\}$, there exists some $f(x, u)$ which satisfies $(2.2)$.

We have shown that if $x \in \mathbb{R}^{n} \backslash\{0\}$ and if the function $V$ is differentiable at $x$, then (2.2) is satisfied with a compact set $W_{x_{i_{0}}}$ which depends on $x$ but which could be taken to be uniform for all $x$ with $\|x\| \leq r$. Let us emphasize that since $\tilde{V}$ is smooth outside the origin and since $V$ is differentiable almost everywhere, our result implies (also by (H1)) that (2.2) is satisfied for any $x \neq 0$. However $V$ is not $C^{\infty}$ at the origin. By Lemma 4.3 in [22], one can find a function $\beta:\left[0, \infty\left[\rightarrow\left[0, \infty\left[\right.\right.\right.\right.$ which is increasing, positive, $C^{\infty}$ on $] 0, \infty[$ and such that the function $\hat{V}(\cdot)=\beta(\tilde{V}(\cdot))$ is $C^{\infty}$ on $\mathbb{R}^{n}$. For this new function, we will have

$$
\partial \hat{V}(x)=\beta^{\prime}(\tilde{V}(x)) \partial \tilde{V}(x) .
$$

Hence it will satisfy the decrease condition (2.2) and will be a smooth control-Lyapunov function.

It remains to prove (ii) $\Longrightarrow$ (iii). Let us denote by $V$ the function given by the assertion (ii). By definition, for any positive integer $i$ there exists a compact set $W_{i}$ such that

$$
\forall y \in 2 i \bar{B} \backslash\{0\}, \min _{u \in W_{i}}\langle\nabla V(y), f(y, u)\rangle<0 .
$$

For each $x \in \mathbb{R}^{n}$, we set $W_{x}:=W_{[x]+1}$ (where $[x]$ denotes the integer part of $\|x\|$ ). Define for any $x \in \mathbb{R}^{n}$ :

$$
h(x):=\inf _{y \in \mathbb{R}^{n}}\left\{-\min _{u \in W_{y}}\langle\nabla V(y), f(y, u)\rangle+\|x-y\|\right\} .
$$

This new function is 1-Lipschitz and positive definite (by (4.28)), and moreover we have by construction:

$$
h(x) \leq-\min _{u \in W_{x}}\langle\nabla V(x), f(x, u)\rangle
$$

This allows us to define for any $x \in \mathbb{R}^{n} \backslash\{0\}$ the following nonempty set:

$$
G(x):=\left\{u \in W_{x}:\langle\nabla V(x), f(x, u)\rangle \leq-h(x)\right\} .
$$


Consider a selection $u(\cdot)$ of $G$ (extended by 0 at the origin). By the good choice of the compact sets $W_{x}$, it is locally bounded. Besides, by linearity of the scalar product, we have for any $x \neq 0$ and for any $v \in K_{u}(x)$,

$$
\langle\nabla V(x), v\rangle \leq-h(x) .
$$

By Theorem 15.1 of [14] we conclude that the differential inclusion

$$
\dot{x}(t) \in K_{u}(x(t)) \quad \text { a.e. }
$$

is globally asymptotically stable at the origin.

Now, if instead of a feedback of Krasovskii type we want to construct a feedback of Filippov type, we have to prove that the multivalued map $K$ which is considered above is measurable. Thus we can extract a measurable selection (see [4]) which will allow us to set the differential inclusion given by Filippov. Then we conclude with the same proof and hence the proof of Theorem 2.7 is complete. We have still to demonstrate Theorem 2.8.

In the case of an affine system, that is when

$$
f(x, u)=f_{0}(x)+\sum_{i=1}^{m} u_{i} f_{i}(x),
$$

we consider the same compact sets $W_{x}$ as above and the same function $h$ given by (4.29). We define for any $x \in \mathbb{R}^{n} \backslash\{0\}:$

$$
K^{\prime}(x):=\left\{u \in W_{x}:\langle\nabla V(x), f(x, u)\rangle \leq-\frac{h(x)}{2}\right\} .
$$

Since the function $f$ is affine in the control, the multivalued map $K^{\prime}$ has nonempty convex compact values. In addition, since $f$ and $\nabla V$ are both continuous and since the sets $G(x)$ given by (4.30) are nonempty, we deduce that the multivalued map $K^{\prime}$ is also lower semicontinuous in $\mathbb{R}^{n} \backslash\{0\}$ (we refer to [13] and [4] for the definition of lower semicontinuity). So, we can apply the famous Michael's theorem (see [24]) and get the existence of a continuous selection $u: \mathbb{R}^{n} \backslash\{0\} \rightarrow U$. Of course, we set $u(0)=0$; by the same proof as for (ii) $\Longrightarrow$ (iii), we get that the dynamical system

$$
\dot{x}=f(x, u(x))
$$

is globally asymptotically stable.

Now if the last assumption of Theorem 2.8 is satisfied, then the function $K^{\prime}$ can be extended to $\mathbb{R}^{n}$ by setting $K^{\prime}(0)=\{0\}$. It is with nonempty convex compact values and furthermore it is henceforth lower semicontinuous in $\mathbb{R}^{n}$. We deduce the existence of a continuous selection on $\mathbb{R}^{n}$ such that $u(0)=0$. Still once we conclude by the proof of (ii) $\Longrightarrow$ (iii) of Theorem 2.7. Theorem 2.8 is proved.

\subsection{Further comments}

In view of the preceding proof, we can easily generalize Theorem 2.7 to the case of stabilization toward a compact set. As a matter of fact, since our proof is mainly based on the compacity of the basin of attraction, we can easily developp all our lemmae in this case. In this way, we obtain some result close to the theorem presented by Praly and Teel in [31].

\section{REFERENCES}

[1] Z. Artstein, Stabilization with relaxed controls. Nonlinear Anal. 7 (1983) 1163-1173.

[2] J.-P. Aubin, Viability theory. Birkhäuser Boston Inc., Boston, MA (1991).

[3] J.P. Aubin and A. Cellina, Differential Inclusions. Springer-Verlag (1984).

[4] J.P. Aubin and H. Frankowska, Set-valued analysis. Birkhäuser (1990).

[5] C.I. Byrnes and A. Isidori, New results and examples in nonlinear feedback stabilization. Systems Control Lett. 12 (1989) 437-442. 
[6] F.H. Clarke, Yu.S. Ledyaev, L. Rifford and R.J. Stern, Feedback stabilization and Lyapunov functions. SIAM J. Control Optim. 39 (2000) 25-48.

[7] F.H. Clarke, Optimization and Nonsmooth Analysis. Wiley-Interscience, New York (1983). Republished as Classics Appl. Math. 5 (1990).

[8] F.H. Clarke, Yu.S. Ledyaev and R.J. Stern, Asymptotic stability and smooth Lyapunov functions. J. Differential Equations 149 (1998) 69-114.

[9] F.H. Clarke, Yu.S. Ledyaev, R.J. Stern and P.R. Wolenski, Nonsmooth Analysis and Control Theory. Springer-Verlag, New York, Grad. Texts in Math. 178 (1998).

[10] J.-M. Coron, On the stabilization of some nonlinear control systems: Results, tools, and applications, in Nonlinear analysis, differential equations and control (Montreal, QC, 1998). Kluwer Acad. Publ., Dordrecht (1999) 307-367.

[11] J.-M. Coron, Some open problems in control theory, in Differential geometry and control (Boulder, CO, 1997). Providence, RI, Amer. Math. Soc. (1999) 149-162.

[12] J.-M. Coron and L. Rosier, A relation between continuous time-varying and discontinuous feedback stabilization. J. Math. Systems Estim. Control 4 (1994) 67-84.

[13] K. Deimling, Multivalued Differential Equations. de Gruyter, Berlin (1992).

[14] A.F. Filippov, Differential Equations with Discontinuous Righthand Sides. Kluwer Academic Publishers (1988).

[15] R. Freeman and P.V. Kokotovic, Robust Nonlinear Control Design. State-Space and Lyapunov Techniques. Birkhäuser (1996).

[16] R.A. Freeman and P.V. Kokotovic, Backstepping design with nonsmooth nonlinearities, in Proc. of the IFAC Nonlinear Control Systems design symposium. Tahoe City, California (1995).

[17] O. Hájek, Discontinuous differential equations. I, II. J. Differential Equations 32 (1979) 149-170, 171-185.

[18] J.-B. Hiriart-Urruty and C. Imbert, Les fonctions d'appui de la jacobienne généralisée de Clarke et de son enveloppe plénière. C. R. Acad. Sci. Paris Sér. I Math. 326 (1998) 1275-1278.

[19] N.N. Krasovskiǔ, Stability of motion. Applications of Lyapunov's second method to differential systems and equations with delay. Stanford University Press, Stanford, California (1963). Translated by J.L. Brenner.

[20] J. Kurzweil, On the inversion of Lyapunov's second theorem on stability of motion. Amer. Math. Soc. Transl. Ser. 2 24 (1956) 19-77.

[21] Yu.S. Ledyaev and E.D. Sontag, A Lyapunov characterization of robust stabilization. Nonlinear Anal. 37 (1999) 813-840.

[22] Y. Lin, E.D. Sontag and Y. Wang, A smooth converse Lyapunov theorem for robust stability. SIAM J. Control Optim. 34 (1996) 124-160.

[23] J.L. Massera, Contributions to stability theory. Ann. of Math. (2) 64 (1956) 182-206.

[24] E. Michael, Continuous selections. I. Ann. of Math. (2) 63 (1956) 361-382.

[25] L. Praly and A.R. Teel, On assigning the derivative of a disturbance attenuation clf, in Proc. of the 3rth IEEE conference on decision and control. Tampa, Florida (1998).

[26] L. Rifford, Existence of Lipschitz and semiconcave control-Lyapunov functions. SIAM J. Control Optim. 39 (2000) $1043-1064$.

[27] L. Rosier, Étude de quelques problèmes de stabilisation, Ph.D. Thesis. ENS de Cachan (1993).

[28] E.D. Sontag, A "universal" construction of Artstein's theorem on nonlinear stabilization. Systems Control Lett. 13 (1989) $117-123$.

[29] E.D. Sontag, Mathematical Control Theory. Springer-Verlag, New York, Texts Appl. Math. 6 (1990) (Second Edition, 1998).

[30] E.D. Sontag, Stability and stabilization: Discontinuities and the effect of disturbances, in Nonlinear analysis, differential equations and control (Montreal, QC, 1998). Kluwer Acad. Publ., Dordrecht (1999) 551-598.

[31] A.R. Teel and L. Praly, A smooth Lyapunov function from a class- $\mathcal{K} \mathcal{L}$ estimate involving two positive semidefinite functions. ESAIM: COCV 5 (2000) 313-367.

[32] J. Tsinias, A Lyapunov description of stability in control systems. Nonlinear Anal. 13 (1989) 3-74.

[33] J. Tsinias, Sufficient Lyapunov-like conditions for stabilization. Math. Control Signals Systems 2 (1989) 343-357.

[34] J. Tsinias, A local stabilization theorem for interconnected systems. Systems Control Lett. 18 (1992) $429-434$.

[35] J. Tsinias, An extension of Artstein's theorem on stabilization by using ordinary feedback integrators. Systems Control Lett. 20 (1993) 141-148. 